\title{
Festschrift in the Honor of Stephen H. White's 70th Birthday
}

\author{
Ana-Nicoleta Bondar • Thomas B. Woolf • \\ Douglas J. Tobias
}

Published online: 30 December 2010

(C) The Author(s) 2010. This article is published with open access at Springerlink.com

\begin{abstract}
The Symposium 'Frontiers in membrane and membrane protein biophysics: experiments and theory', held this year at the University of California, Irvine (August 19-20), celebrated the 70th Birthday of Stephen $\mathrm{H}$. White by bringing together distinguished experimentalists and theoreticians to discuss the state of the art and future challenges in the field of membrane and membrane protein biophysics. The meeting and this special issue highlight the highly interdisciplinary nature of membrane and membrane protein biophysics, and the tremendous contributions that S. H. White and his lab have brought to the field.
\end{abstract}

Keywords S. H. White - Membrane and membrane protein biophysics

The lipid membranes surrounding the cell and its various compartments, and the proteins incorporated into the lipid membranes, are of fundamental importance for the survival of a biological cell. Membrane proteins communicate with the lipid membranes to mediate physiological processes

\section{A.-N. Bondar $(\square)$}

Department of Physics, Freie Universität Berlin,

Arnimallee 14, 14195 Berlin-Dahlem, Germany

e-mail: nbondar@zedat.fu-berlin.de

\section{T. B. Woolf $(\bowtie)$}

Department of Physiology, School of Medicine, Johns Hopkins University, 204 Biophysics Building, 725 N. Wolfe Street,

Baltimore, MD 21205, USA

e-mail: tbw.jhmi@gmail.com

D. J. Tobias $(\bowtie)$

Department of Chemistry, University of California,

Irvine, Irvine, CA 92697-2025, USA

e-mail:dtobias@uci.edu such as transport of ions, drugs, nutrients, or other proteins, recognition of neurotransmitters, or perception of light and other stimuli. The properties of the complex lipid mixtures required for the functioning of specific membrane proteins, how membrane proteins are inserted into the lipid membranes, and how the lipid membrane environment influence the structure and dynamics of membrane proteins, are key open issues. The lab of S. H. White at the University of California, Irvine, is among the labs that have paved the way and led the on-going march of progress in the field. On the occasion of Stephen H. White's 70th Birthday, some of us (A.-N. B. and D.J. T.) organized a two-days symposium with the generous support of the Department of Physiology and Biophysics, the Department of Chemistry, the Office of Research, and the Center for Biomembrane Systems of the University of California, Irvine. It was an exciting meeting that brought together distinguished and junior scientists that use state-of-the-art experimental and theoretical techniques to pursue challenging problems in membrane and membrane protein biophysics.

The introductory words were given by Prof. Michael Cahalan, Chairman of the Department of Physiology and Biophysics, Prof. Paolo Casali, Senior Associate Dean for Research and Graduate Studies at UC Irvine School of Medicine, and D.J.T., Professor in the Department of Chemistry of UC Irvine. Throughout the Symposium, speakers and chairpersons, many of them with close scientific ties to Stephen H. White, touched upon the pioneering work of Stephen $\mathrm{H}$. White, his vision, high scientific standards, and remarkable reputation in the field.

Stephen H. White (Steve for his close collaborators and friends) was born in Wewoka, Oklahoma. He studied Physics at the University of Colorado (B.A., 1963). While at the Physics Department of the University of Colorado, 
Steve worked as a technician in the NMR lab of Prof. Walter Tantilla, helped develop a speech synthesizing system in the lab of Prof. D. G. Burkhardt, and did independent research in the lab of Prof. M. Mizushima on a liquid-state laser using aromatic hydrocarbons as active materials. For his M.S. in Physics (1965), Steve studied at the University of Washington. There, he worked with Prof. H.G. Dehmelt (Department of Physics) on the radio frequency spectrum of the $\mathrm{H}_{2}{ }^{+}$ion. In 1969, Steve obtained his PhD degree in Physiology and Biophysics for his work in the lab of Prof. J. Woodbury (University of Washington). His dissertation was entitled 'A Study of the Specific Capacitance of Black Lipid Membranes'. Upon completion of his PhD, Steve served two years in the US Army Chemical Corps to the rank of Captain (1969-1971). From 1971 to 1972 , Steve was a U.S. Public Health Service Postdoctoral Fellow, sponsored by Prof. T.E. Thomson in the Department of Chemistry, University of Virginia School of Medicine.

At age 32, Steve joined the Department of Physiology and Biophysics of the University of California, Irvine as an Assistant Professor, and was promoted to Associate Professor in 1975, and Professor in 1979. Steve made known his independent thinking and extremely high scientific standards from the very beginning of his outstanding career: while still a student and then, later, as young Assistant and Associate Professor, Steve published a series of single-author original articles and commentaries in the Biophysical Journal (1970, 1972, 1974, 1975, 1978), Biochimica and Biophysica Acta (1973, 1974), and Nature (1976). These were the first of long succession of highimpact publications on the physical-chemical properties of fluid lipid bilayers that established the foundation of the Steve's influential work on fundamental aspects of membrane protein folding, assembly, and function.

In addition to his research, Steve also served on numerous advisory boards and University committees, including Vice-Chairman of the Department of Physiology and Biophysics at UC Irvine between 1974 and 1975, and Chairman from October 1977 to March 1989. He served as President of the Biophysical Society in 1996-1997, and as the electronic publishing coordinator for Protein Science during 1992-2008. For his outstanding contributions to science and teaching Steve received numerous awards and honors, including the Kaiser-Permanente Award for Excellence in Teaching in 1975 and 1992, and, more recently, the $\mathrm{PhD}$ honoris causa from the Stockholm University (2008), and the Avanti Award in Lipids (Biophysical Society, 2009) "for his novel findings in the areas of membrane structure and protein insertion into membranes". Steve received the Biophysical Society Distinguished Service Award in 1999, and was elected a Biophysical Society Fellow in 2002.
In his lab, Steve's students and collaborators benefit tremendously from a distinctive synergy of experimental and theoretical studies of membrane and membrane proteins. Indeed, working with Steve is a privilege. He cares deeply about the science pursued in the lab, and about the scientific development of the junior researchers in his lab.

The topics of the Symposium were at the interface between physical chemistry, biology, chemistry, and computational biophysics, and included sessions on the biophysics of lipid:lipid and lipid:protein interactions (Melanie Cocco, Kalina Hristova, Alexey Ladokhin, Eric Lindner, Francesco Tombola, Martin Ulmschneider, Xi Wang), theoretical membrane and membrane protein biophysics (Ioan Andricioaei, Alfredo Freites, Klaus Schulten, Thomas B. Woolf), the structure and the function of membrane proteins (Michael Cahalan, Karen M. Fleming, Jim Hall, Ron Kaback, Janos K. Lanyi, Hartmut Luecke, Daniel Mueller, Ray Stevens, Bob Stroud, Michael C. Wiener), ion channels and nanopores (Zuzana Siwy, Kenton Swartz, David Worcester), and on how membrane proteins get inserted into the lipid membrane (Jim Bowie, Gunnar von Heijne, Venkatramanan Krishmani, William C. Wimley). The schedule of the meeting, and a photo gallery, can be found under http://blanco.biomol.uci.edu/ index.shtml\#labMembers (photos courtesy of Prof. Janos K. Lanyi). We thank all participants at the S. H. White Symposium for joining us in celebrating Steve's Birthday. We also thank the members of Steve White's and D.J.T.'s labs and the UC Irvine staff who helped us organize the Symposium.

We are most grateful to the authors who submitted their work for publication in the S. H. White Festchrift, and to the Editorial Board of the Journal of Membrane Biology for hosting the special issue. The Festschrift covers a wide range of topics in membrane and membrane protein biophysics.

Nguyen et al. studied the binding of single-stranded DNA molecules to synthetic nanopores, and found that the effective diameter of the nanopore can be tuned by changing the ionic strength. This work sets the foundation for systematic studies of DNA-modified nanopores.

R. Kaback et al., E. Imasheva et al., and Bondar et al., investigate the functioning of membrane proteins using experiments (R. Kaback et al.; E. Imasheva et al.) or computer simulations (A.-N. Bondar et al.). The LacY symporter is the prototype of the Major Facilitator Superfamily of membrane transport mechanism; R. Kaback et al. review the biochemical and biophysical evidence supporting the alternating access mechanism of substrate translocation.

By removing and then reconstituting the carotenoid antenna of the xanthorhodopsin proton pump, Imasheva et al. show that removing the carotenoid (salinixanthin) has 
little effect on the kinetics of the photocycle, and that the carbonyl group in the carotenoid ring is necessary for binding of the carotenoid and for efficient energy transfer. A.-N. Bondar et al. use computer simulations to address the question of whether a critical water molecule could change its location during the proton-pumping cycle of bacteriorhodopsin.

A. Frank and I. Andricioaei tested the accuracy of the implicit membrane model 1 (IMM1) and of the Generalized Born with a Simple Switching (GBSW) model in describing the tilt angles of a transmembrane helix of Vpu, an accessory protein of HIV-1. They find that both models give reasonable agreement with the experimental data.

M. Mihailescu et al. report on neutron diffraction and NMR experiments to assess the structure and dynamics of cholesterol-containing polyunsaturated lipid membranes; understanding how cholesterol interacts with polyunsaturated lipids is important, because changes in the lipid organization due to the presence of cholesterol can affect membrane protein function.

Ulmschneider et al. review computer simulations for determining the properties of peptide partitioning into model lipid bilayers. The question of membrane partitioning was also addressed by M. Fernández-Vidal et al.; their experimental observations lead to the conclusion that binding of peptides to lipid membranes is driven by the classical hydrophobic effect.

The role and the interactions of Arg amino acids present in transmembrane bilayers has been under intense scrutiny during the past several years. K. Hristova and W.C. Wimley take 'a look at arginine in membranes' and give a critical discussion of experimental and theoretical studies on the energetics of inserting Arg into a lipid bilayer core. Arginines in membranes are also the focus of the paper by Schow et al., which reports new computer simulations and an in-depth analysis of experimental and theoretical findings that is expected to help clarify the longstanding discrepancy between simulations and experiments on the energetics of Arg in membranes.

Open Access This article is distributed under the terms of the Creative Commons Attribution Noncommercial License which permits any noncommercial use, distribution, and reproduction in any medium, provided the original author(s) and source are credited. 